\title{
MOORE-PENROSE INVERSE OF SOME LINEAR MAPS ON INFINITE-DIMENSIONAL VECTOR SPACES*
}

\author{
VÍCTOR CABEZAS SÁNCHEZ† AND FERNANDO PABLOS ROMO
}

\begin{abstract}
The aim of this work is to characterize linear maps of infinite-dimensional inner product spaces where the Moore-Penrose inverse exists. This MP inverse generalizes the well-known Moore-Penrose inverse of a matrix $A \in$ Mat $n \times m(\mathbb{C})$. Moreover, a method for the computation of the MP inverse of some endomorphisms on infinite-dimensional vector spaces is given. As an application, the least norm solution of an infinite linear system from the Moore-Penrose inverse offered is studied.
\end{abstract}

Key words. Infinite-dimensional vector space, Moore-Penrose inverse, Infinite linear system.

AMS subject classifications. 34A30, 15A06, 15A04.

1. Introduction. Given a matrix $A \in \operatorname{Mat}_{n \times m}(\mathbb{C})$, the Moore-Penrose inverse of $A$ is the unique matrix $A^{\dagger} \in \operatorname{Mat}_{m \times n}(\mathbb{C})$ such that:

- $A A^{\dagger} A=A$;

- $A^{\dagger} A A^{\dagger}=A^{\dagger}$;

- $\left(A A^{\dagger}\right)^{*}=A A^{\dagger}$

- $\left(A^{\dagger} A\right)^{*}=A^{\dagger} A$;

where $B^{*}$ is the conjugate transpose of the matrix $B$.

The Moore-Penrose inverse of $A$ always exists, it is a reflexive generalized inverse of $A,\left[A^{\dagger}\right]^{\dagger}=A$ and, if $A \in \operatorname{Mat}_{n \times n}(\mathbb{C})$ is non-singular, then $A^{\dagger}$ coincides with the inverse matrix $A^{-1}$ (for details, readers are referred to [2]).

Recently, the notion of generalized inverses of matrices $A \in \operatorname{Mat}_{n \times m}(\mathbb{C})$ has been extended to some linear maps on infinite-dimensional vector spaces. Indeed, the authors have computed explicit solutions of infinite systems of linear equations from reflexive generalized inverses of finite potent endomorphisms in [3] and, also, the second-named author has generalized the notion of Drazin inverse to finite potent endomorphisms in [4].

The aim of this work is to characterize linear maps of infinite-dimensional inner product spaces where the Moore-Penrose (MP) inverse exists. This MP inverse generalizes the Moore-Penrose inverse $A^{\dagger}$ of a matrix $A \in \operatorname{Mat}_{n \times m}(\mathbb{C})$. Moreover, a method for the computation of the MP inverse of some endomorphisms on infinite-dimensional vector spaces is given. As an application, we study the least norm solution of an infinite linear system from this Moore-Penrose inverse.

${ }^{*}$ Received by the editors on October 10, 2018. Accepted for publication on June 22, 2020. Handling Editor: Bryan L. Shader. Corresponding Author: Fernando Pablos Romo.

${ }^{\dagger}$ Departamento de Matemáticas, Universidad de Salamanca, Plaza de la Merced 1-4, 37008 Salamanca, España (v.cabezas@usal.es). Partially supported by a collaboration-fellowship of the Spanish Government.

¥Departamento de Matemáticas and Instituto de Física Fundamental y Matemáticas, Universidad de Salamanca, Plaza de la Merced 1-4, 37008 Salamanca, España (fpablos@usal.es). Partially supported by the Spanish Government research projects no. MTM2015-66760-P and no. PGC2018-099599-B-I00, and the Regional Government of Castile and Leon research project no. J416/463AC03. 
The paper is organized as follows. In Section 2, we recall the basic definitions of this work: inner product vector spaces, finite potent endomorphisms, reflexive generalized inverse and Moore-Penrose inverse of a $(n \times m)$-matrix. Also, in this section, we briefly describe the construction of Jordan bases for endomorphisms admitting an annihilator polynomial.

Section 3 contains the main results the this work: the definition of linear map admissible for the MoorePenrose inverse (Definition 3.10), the proof of the existence and uniqueness of the MP inverse for these linear maps (Theorem 3.12) and the conditions for computing the MP inverse for some endomorphisms on infinite-dimensional vector spaces from the MP inverses of $(n \times n)$-matrices (Theorem 3.20).

Finally, Section 4 is devoted to study infinite systems of linear equations from the Moore-Penrose Inverse. Thus, Proposition 4.4 shows that if $(V, g)$ and $(W, \bar{g})$ are two arbitrary inner product vector spaces over $\mathbb{R}$ or $\mathbb{C}, f: V \rightarrow W$ is a linear map admissible for the Moore-Penrose inverse and $f(x)=w$ is a linear system, then $f^{\dagger}(w)$ is the unique minimal least $\bar{g}$-norm solution of this linear system.

2. Preliminaries. This section is added for the sake of completeness.

2.1. Inner product vector spaces. Let $k$ be the field of the real numbers or the field of the complex numbers, and let $V$ be a $k$-vector space.

An inner product on $V$ is a map $g: V \times V \rightarrow k$ satisfying:

- $g$ is linear in its first argument:

$$
g\left(\lambda v_{1}+\mu v_{2}, v^{\prime}\right)=\lambda g\left(v_{1}, v^{\prime}\right)+\mu g\left(v_{2}, v^{\prime}\right) \quad \text { for every } \quad v_{1}, v_{2}, v^{\prime} \in V
$$

- $g\left(v^{\prime}, v\right)=\overline{g\left(v, v^{\prime}\right)}$ for all $v, v^{\prime} \in V$, where $\overline{g\left(v, v^{\prime}\right)}$ is the complex conjugate of $g\left(v, v^{\prime}\right)$;

- $g$ is positive definite:

$$
g(v, v) \geq 0 \quad \text { and } \quad g(v, v)=0 \Longleftrightarrow v=0 .
$$

Note that $g(v, v) \in \mathbb{R}$ for each $v \in V$, because $g(v, v)=\overline{g(v, v)}$.

An inner product space is a pair $(V, g)$.

If $(V, g)$ is an inner product vector space over $\mathbb{C}$, it is clear that $g$ is antilinear in its second argument, that is:

$$
g\left(v, \lambda v_{1}^{\prime}+\mu v_{2}^{\prime}\right)=\bar{\lambda} g\left(v, v_{1}^{\prime}\right)+\bar{\mu} g\left(v, v_{2}^{\prime}\right)
$$

for all $v, v_{1}^{\prime}, v_{2}^{\prime} \in V$, and $\bar{\lambda}$ and $\bar{\mu}$ being the conjugates of $\lambda$ and $\mu$, respectively.

Nevertheless, if $(V, \mathrm{~g})$ is an inner product vector space over $\mathbb{R}$, then $g$ is symmetric and bilinear.

The norm on an inner product vector space $(V, g)$ is the real-valued function

$$
\begin{aligned}
\|\cdot\|_{g}: V & \longrightarrow \mathbb{R} \\
v & \longmapsto+\sqrt{g(v, v),}
\end{aligned}
$$

and the distance is the map

$$
\begin{aligned}
d_{g}: V \times V & \longrightarrow \mathbb{R} \\
\left(v, v^{\prime}\right) & \longmapsto\left\|v^{\prime}-v\right\|_{g} .
\end{aligned}
$$


Simple examples of inner product vector spaces are Euclidean finite-dimensional real vector spaces and complex Hilbert spaces.

Let us now consider two inner product vector spaces: $(V, g)$ and $(W, \bar{g})$. If $f: V \rightarrow W$ is a linear map, a linear operator $f^{*}: W \rightarrow V$ is called the adjoint of $f$ when

$$
g\left(f^{*}(w), v\right)=\bar{g}(w, f(v))
$$

for all $v \in V$ and $w \in W$. If $f \in \operatorname{End}_{k}(V)$, we say that $f$ is self-adjoint when $f^{*}=f$.

Moreover, if $(V, g)$ and $(W, \bar{g})$ are finite-dimensional inner vector spaces over $\mathbb{C}, B=\left\{v_{1}, \ldots, v_{m}\right\}$ and $B^{\prime}=\left\{w_{1}, \ldots, w_{n}\right\}$ are orthonormal bases of $V$ and $W$, respectively, $f: V \rightarrow W$ is a linear map, $A \in \operatorname{Mat}_{n \times m}(\mathbb{C})$ and $f \equiv A$ in these bases, then $f^{*} \equiv A^{*} \in \operatorname{Mat}_{m \times n}(\mathbb{C})$ in the same bases, where $A^{*}$ is the conjugate transpose of $A$.

2.2. Jordan bases for endomorphisms admitting an annihilator polynomial. Let $V$ be an arbitrary vector space over a ground field $k$, and let $f \in \operatorname{End}_{k}(V)$ be an endomorphism of $V$ admitting an annihilator polynomial

$$
a_{f}(x)=p_{1}(x)^{n_{1}} \cdot p_{r}(x)^{n_{r}},
$$

where $p_{i}(x)$ are irreducible polynomials in $k[x]$ and $n_{i}$ are positive integers.

For each $j \in\{1, \ldots, r\}$, we can consider

$$
\nu_{i}\left(V, p_{j}(f)\right)=\operatorname{dim}_{K_{j}}\left(\operatorname{Ker} p_{j}(f)^{i} /\left[\operatorname{Ker} p_{j}(f)^{i-1}+p_{j}(f) \operatorname{Ker} p(f)^{i+1}\right]\right),
$$

with $K_{j}=k[x] / p_{j}(x)$. Henceforth, $S_{\nu_{i}\left(V, p_{j}(f)\right)}$ will be a set such that $\# S_{\nu_{i}\left(V, p_{j}(f)\right)}=\nu_{i}\left(V, p_{j}(f)\right)$, with $S_{\nu_{i}\left(V, p_{j}(f)\right)} \cap S_{\nu_{h}\left(V, p_{j}(f)\right)}=\emptyset$ for $i \neq h$.

According to the statements of [5], there exist families of vectors $\left\{v_{h}^{i j}\right\}_{h \in S_{\nu_{i}\left(V, p_{j}(f)\right)}}$ with

$$
v_{h}^{i j} \in \operatorname{Ker} p_{j}(f)^{i} \quad \text { and } \quad v_{h}^{i j} \notin \operatorname{Ker} p_{j}(f)^{i-1}+p_{j}(f) \operatorname{Ker} p_{j}(f)^{i+1}
$$

for all $1 \leq j \leq r$ and $1 \leq i \leq n_{j}$, such that if we set

$$
H_{h}^{i j}=<v_{h}^{i j}>_{f}=\bigcup_{0 \leq s \leq i-1}\left\{p_{j}(f)^{s}\left[v_{h}^{i j}\right], p_{j}(f)^{s}\left[f\left(v_{h}^{i j}\right)\right], \ldots, p_{j}(f)^{s}\left[f^{d_{j}-1}\left(v_{h}^{i j}\right)\right]\right\},
$$

where $d_{j}=\operatorname{dim}_{k} K_{j}$, then

$$
\begin{aligned}
& \bigcup_{1 \leq j \leq r}<v_{h}^{i j}>_{f} \\
& 1 \leq i \leq n_{j} \\
& h \in S_{\nu_{i}\left(V, p_{j}(f)\right)}
\end{aligned}
$$

is a Jordan basis of $V$ for $f$, and this basis determines a decomposition

$$
\begin{aligned}
& V=\underset{1 \leq j \leq r}{\bigoplus} H_{h}^{i j} . \\
& 1 \leq i \leq n_{j} \\
& h \in S_{\nu_{i}\left(V, p_{j}(f)\right)}
\end{aligned}
$$


EXAMPLE 2.1. (Jordan bases for a nilpotent endomorphism) Let $V$ be a vector space over an arbitrary field $k$ and let $f \in \operatorname{End}_{k}(V)$ be a nilpotent endomorphism. If $f$ has index of nilpotency n, setting $W_{i}^{f}=$ $\operatorname{Ker} f^{i} /\left[\operatorname{Ker} f^{i-1}+f\left(\operatorname{ker} f^{i+1}\right)\right]$ with $i \in\{1,2, \ldots, n\}, \mu_{i}(V, f)=\operatorname{dim}_{k} W_{i}^{f}$ and $S_{\mu_{i}(V, f)}$ a set such that $\# S_{\mu_{i}(V, f)}=\mu_{i}(V, f)$ with $S_{\mu_{i}(V, f)} \cap S_{\mu_{j}(V, f)}=\emptyset$ for all $i \neq j$, one has that there exists a family of vectors $\left\{v_{s_{i}}\right\}$ that determines a Jordan basis of $V$ for $f$ :

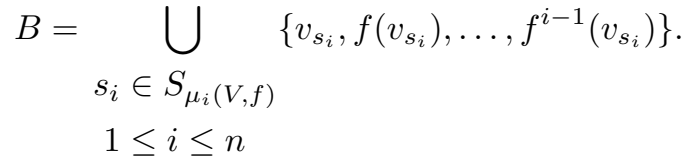

Moreover, if we write $H_{s_{i}}^{f}=\left\langle v_{s_{i}}, f\left(v_{s_{i}}\right), \ldots, f^{i-1}\left(v_{s_{i}}\right)\right\rangle$, the basis $B$ induces a decomposition

$$
\begin{gathered}
V=\bigoplus_{s_{i} \in S_{\mu_{i}(V, f)}} H_{s_{i}}^{f} \\
1 \leq i \leq n
\end{gathered}
$$

2.3. Finite potent endomorphisms. Let $k$ be an arbitrary field, let $V$ be a $k$-vector space and let $\varphi \in \operatorname{End}_{k}(V)$. We say that $\varphi$ is "finite potent" if $\varphi^{n} V$ is finite dimensional for some $n$. This definition was introduced by J. Tate in [7] as a basic tool for his elegant definition of Abstract Residues.

In 2007 M. Argerami, F. Szechtman and R. Tifenbach showed in [1] that an endomorphism $\varphi$ is finite potent if and only if $V$ admits a $\varphi$-invariant decomposition $V=U_{\varphi} \oplus W_{\varphi}$ such that $\varphi_{\left.\right|_{\varphi}}$ is nilpotent, $W_{\varphi}$ is finite dimensional, and $\varphi_{\left.\right|_{\varphi}}: W_{\varphi} \stackrel{\sim}{\longrightarrow} W_{\varphi}$ is an isomorphism.

2.4. Reflexive generalized inverses. Let $\mathbb{C}$ be the field of complex numbers. Given a matrix $A \in$ $\operatorname{Mat}_{n \times m}(\mathbb{C})$, a reflexive generalized inverse of $A$ is a matrix $A^{+} \in \operatorname{Mat}_{m \times n}(\mathbb{C})$ such that:

- $A A^{+} A=A$;

- $A^{+} A A^{+}=A^{+}$.

In general, the reflexive generalized inverse of a matrix $A$ is not unique.

The notion of reflexive generalized inverse in arbitrary vector spaces is the following:

Definition 2.2. If $V$ and $W$ are $k$-vector spaces, given a morphism $f: V \rightarrow W$, a linear map $f^{+}: W \rightarrow V$ is a "reflexive generalized inverse" of $f$ when:

- $f \circ f^{+} \circ f=f$

- $f^{+} \circ f \circ f^{+}=f^{+}$.

For every reflexive generalized inverse $f^{+}$of $f$, if $w \in W$, it is known that

$$
w \in \operatorname{Im} f \Longleftrightarrow\left(f \circ f^{+}\right)(w)=w .
$$

3. Moore-Penrose inverse of linear maps on infinite-dimensional vector spaces. This section is devoted to proving the existence of a Moore-Penrose inverse of some linear maps on infinite-dimensional vector spaces, such that it generalizes the notion and the properties of the Moore-Penrose inverse of an $(n \times m)$-matrix with entries in $\mathbb{C}$. Our generalization will be valid for linear maps on inner product vector 
spaces over $k=\mathbb{R}$ and $k=\mathbb{C}$ and we shall give conditions for computing the Moore-Penrose inverse of endomorphisms on infinite-dimensional vector spaces.

To do so, we shall first study some properties of the Moore-Penrose inverse of an endomorphism on a finite-dimensional inner product space.

3.1. Moore-Penrose inverse of an endomorphism on finite-dimensional inner product vector spaces. Let us now consider a finite dimensional inner product vector space $(E, g)$ over $k=\mathbb{R}$ or $k=\mathbb{C}$.

If $f \in \operatorname{End}_{k}(E)$, one has that $E=\operatorname{Ker} f \oplus[\operatorname{Ker} f]^{\perp}=\operatorname{Im} f \oplus[\operatorname{Im} f]^{\perp}$, and there exists an isomorphism

$$
f:[\operatorname{Ker} f]^{\perp} \stackrel{\sim}{\longrightarrow} \operatorname{Im} f .
$$

Thus, the Moore-Penrose of $f$ is the unique linear map $f^{\dagger} \in \operatorname{End}_{k}(E)$ such that

$$
f^{\dagger}(e)=\left\{\begin{array}{cll}
\left(f_{\left.\right|_{[\operatorname{Ker} f]^{\perp}}}\right)^{-1}(e) & \text { if } & e \in \operatorname{Im} f \\
0 & \text { if } & e \in[\operatorname{Im} f]^{\perp}
\end{array} .\right.
$$

It is known that $f^{\dagger}$ is the unique linear map such that:

- $f^{\dagger}$ is a reflexive generalized inverse of $f$;

- $f^{\dagger} \circ f$ and $f \circ f^{\dagger}$ are self-adjoint.

Definition 3.1. If $f \in \operatorname{End}_{k}(E)$ and $\mathcal{H}_{f}=\left\{H_{1}, \ldots, H_{n}\right\}$ is a family of subspaces of $E$ invariants for $f$ such that $E=H_{1} \oplus \cdots \oplus H_{n}$, we define the endomorphism $f_{\mathcal{H}_{f}}^{+} \in \operatorname{End}_{k}(E)$ as the unique linear map such that

$$
\left[f_{\mathcal{H}_{f}}^{+}\right]_{\left.\right|_{H_{i}}}=\left[f_{\left.\right|_{H_{i}}}\right]^{\dagger} \quad \text { for each } i \in\{1, \ldots, n\} \text {. }
$$

If we denote $f_{i}=f_{\left.\right|_{H_{i}}}$ for each $i \in\{1, \ldots, n\}$, it is clear that for every vector $e \in E$, such that $e=h_{j_{1}}+\cdots+h_{j_{n}}$ with $h_{j_{i}} \in H_{i}$, then

$$
f_{\mathcal{H}_{f}}^{+}(e)=f_{1}^{\dagger}\left(h_{j_{1}}\right)+\cdots+f_{n}^{\dagger}\left(h_{j_{n}}\right) .
$$

Moreover, it is immediately observed, from Definition 3.1 and from the properties of the Moore-Penrose inverse, that $f_{\mathcal{H}_{f}}^{+}$is a reflexive generalized inverse of $f$ for every family $\mathcal{H}_{f}$.

Keeping the previous notation and given a subspace $W \subseteq E$, such that $W \subset H_{i}$ for a certain $i \in$ $\{1, \ldots, n\}$, we shall denote

$$
W_{i}^{\perp}=\left\{v_{i} \in H_{i} \text { such that } g\left(w, v_{i}\right)=0 \text { for all } w \in W\right\} .
$$

LEMma 3.2. If $U \subset E$ is a subspace and $\left\{U_{1}, \ldots, U_{n}\right\}$ are subspaces of $U$ such that $U=U_{1} \oplus \cdots \oplus U_{n}$ with $U_{i} \subseteq H_{i}$, then

$$
\left[U_{1}\right]_{1}^{\perp} \oplus \cdots \oplus\left[U_{n}\right]_{n}^{\perp} \subseteq U^{\perp} \quad \text { if and only if } \quad\left[U_{i}\right]_{i}^{\perp} \subset\left[\sum_{j \neq i} U_{j}\right]^{\perp} \text { for all } i \in\{1, \ldots, n\} .
$$

Proof. If $\left[U_{1}\right]_{1}^{\perp} \oplus \cdots \oplus\left[U_{n}\right]_{n}^{\perp} \subseteq U^{\perp}$, then $\left[U_{i}\right]_{i}^{\perp} \subseteq U^{\perp}$ for all $i \in\{1, \ldots, n\}$ and we deduce that $\left[U_{i}\right]_{i}^{\perp} \subseteq\left[\sum_{j \neq i} U_{j}\right]^{\perp}$ for all $i \in\{1, \ldots, n\}$. 
Conversely, if $\left[U_{i}\right]_{i}^{\perp} \subseteq\left[\sum_{j \neq i} U_{j}\right]^{\perp}$ for all $i \in\{1, \ldots, n\}$, one has that

$$
g\left(v_{1}+\cdots+v_{n}, u_{1}+\cdots+u_{n}\right)=0
$$

with $v_{i} \in\left[U_{i}\right]_{i}^{\perp}$ and $u_{i} \in U_{i}$ for all $i \in\{1, \ldots, n\}$ and, therefore, we obtain that $\left[U_{1}\right]_{1}^{\perp} \oplus \cdots \oplus\left[U_{n}\right]_{n}^{\perp} \subseteq U^{\perp} . \square$

LEMMA 3.3. Using the previous notation, we have that:

1. $\operatorname{Im} f=\operatorname{Im} f_{1} \oplus \cdots \oplus \operatorname{Im} f_{n}$;

2. If $\mathcal{H}_{f}^{\perp}=\left[\operatorname{Im} f_{1}\right]_{1}^{\perp} \oplus \cdots \oplus\left[\operatorname{Im} f_{n}\right]_{n}^{\perp}$, then $E=\operatorname{Im} f \oplus \mathcal{H}_{f}^{\perp}$;

3. $\operatorname{Ker} f=\operatorname{Ker} f_{1} \oplus \cdots \oplus \operatorname{Ker} f_{n}$;

4. If $\widetilde{\mathcal{H}}_{f}^{\perp}=\left[\operatorname{Ker} f_{1}\right]_{1}^{\perp} \oplus \cdots \oplus\left[\operatorname{Ker} f_{n}\right]_{n}^{\perp}$, then

$$
E=\operatorname{Ker} f \oplus \widetilde{\mathcal{H}}_{f}^{\perp}
$$

5. $f$ induces an isomorphism between $\widetilde{\mathcal{H}}_{f}^{\perp}$ and $\operatorname{Im} f$ and

$$
\left[f_{\mathcal{H}_{f}}^{+}\right](e)=\left\{\begin{array}{cl}
\left(f_{\left.\right|_{\widetilde{\mathcal{H}}_{f}^{\perp}}}\right)^{-1}(e) & \text { if } e \in \operatorname{Im} f \\
0 & \text { if } e \in \mathcal{H}_{f}^{\perp}
\end{array} .\right.
$$

Proof. 1. It is clear that $\operatorname{Im} f_{1} \oplus \cdots \oplus \operatorname{Im} f_{n} \subseteq \operatorname{Im} f$. Moreover, given $e \in \operatorname{Im} f$, if $e=f\left(e^{\prime}\right)$ with $e^{\prime}=e_{j_{1}}^{\prime}+\cdots+e_{j_{n}}^{\prime}$ and $e_{j_{i}}^{\prime} \in H_{i_{j}}$ for all $i \in\{1, \ldots, n\}$, then

$$
e=f\left(e_{j_{1}}^{\prime}\right)+\cdots+f\left(e_{j_{n}}^{\prime}\right) \in \operatorname{Im} f_{1} \oplus \cdots \oplus \operatorname{Im} f_{n}
$$

because $f\left(v_{j_{i}}^{\prime}\right) \in H_{i}$ for every $i \in\{1, \ldots, n\}$.

2. Since $H_{i}=\operatorname{Im} f_{i} \oplus\left[\operatorname{Im} f_{i}\right]_{i}^{\perp}$ for all $i \in\{1, \ldots, n\}$, we have that

$$
\begin{aligned}
E & =H_{1} \oplus \cdots \oplus H_{n}=\left(\operatorname{Im} f_{1} \oplus\left[\operatorname{Im} f_{1}\right]_{1}^{\perp}\right) \oplus \cdots \oplus\left(\operatorname{Im} f_{n} \oplus\left[\operatorname{Im} f_{n}\right]_{n}^{\perp}\right) \\
& =\left(\operatorname{Im} f_{1} \oplus \cdots \oplus \operatorname{Im} f_{n}\right) \oplus\left(\left[\operatorname{Im} f_{1}\right]_{1}^{\perp} \oplus \cdots \oplus\left[\operatorname{Im} f_{n}\right]_{n}^{\perp}\right)=\operatorname{Im} f \oplus \mathcal{H}_{f}^{\perp} .
\end{aligned}
$$

3. It is immediate that $\operatorname{Ker} f_{1} \oplus \cdots \oplus \operatorname{Ker} f_{n} \subseteq \operatorname{Ker} f$. Furthermore, if $\bar{e} \in \operatorname{Ker} f$ and

$$
\bar{e}=\bar{e}_{j_{1}}+\cdots+\bar{e}_{j_{n}} \quad \text { with } \quad \bar{e}_{j_{i}} \in H_{i} \quad \text { for every } i \in\{1, \ldots, n\},
$$

since

$$
f\left(\bar{e}_{j_{1}}\right)+\cdots+f\left(\bar{e}_{j_{k}}\right)=0 \quad \text { and } \quad H_{i} \cap\left[\sum_{r \neq i} H_{r}\right]=0
$$

we conclude that $f\left(\bar{e}_{j_{i}}\right)=0$ for all $i \in\{1, \ldots, n\}$, from which it is deduced that $\operatorname{Ker} f \subseteq \operatorname{Ker} f_{1} \oplus \cdots \oplus \operatorname{Ker} f_{n}$.

4. Similar to (2).

5. It is a direct consequence of $(2),(4)$ and the definition of $f_{\mathcal{H}_{f}}^{+}$.

REMARK 3.4. With the previous notation, in general $\widetilde{\mathcal{H}}_{f}^{\perp} \neq[\operatorname{Ker} f]^{\perp}$ and $\mathcal{H}_{f}^{\perp} \neq[\operatorname{Im} f]^{\perp}$, which is deduced from the following counter-example.

Let $E=\left\langle v_{1}, v_{2}, v_{3}, v_{4}\right\rangle$ be a inner product $k$-vector space, $B=\left\{v_{1}, v_{2}, v_{3}, v_{4}\right\}$ being an orthonormal basis, and let us consider the endomorphism $f: E \rightarrow E$ defined as:

$$
f\left(v_{i}\right)=\left\{\begin{array}{ccc}
v_{1} & \text { if } & i=1,2 \\
-2 v_{1} & \text { if } & i=3 \\
v_{1}+v_{2}+v_{3} & \text { if } & i=4
\end{array} .\right.
$$


If we set $H_{1}=\left\langle v_{1}, v_{2}\right\rangle$ and $H_{2}=\left\langle v_{1}+v_{2}+v_{3}, v_{4}\right\rangle$, it is clear that $H_{1}$ and $H_{2}$ are $f$-invariant and, if we again denote $f_{1}=f_{\left.\right|_{H_{1}}}$ and $f_{2}=f_{\left.\right|_{H_{2}}}$, we have that:

- $\operatorname{Im} f_{1}=\left\langle v_{1}\right\rangle$ and $\left[\operatorname{Im} f_{1}\right]_{1}^{\perp}=\left\langle v_{2}\right\rangle$;

- $\operatorname{Im} f_{2}=\left\langle v_{1}+v_{2}+v_{3}\right\rangle$ and $\left[\operatorname{Im} f_{2}\right]_{2}^{\perp}=\left\langle v_{4}\right\rangle$;

- $\operatorname{Im} f=\left\langle v_{1}, v_{1}+v_{2}+v_{3}\right\rangle$ and $[\operatorname{Im} f]^{\perp}=\left\langle v_{2}-v_{3}, v_{4}\right\rangle$;

- Ker $f_{1}=\left\langle v_{1}-v_{2}\right\rangle$ and $\left[\operatorname{Ker} f_{1}\right]_{1}^{\perp}=\left\langle v_{1}+v_{2}\right\rangle$;

- $\operatorname{Ker} f_{2}=\left\langle v_{1}+v_{2}+v_{3}\right\rangle$ and $\left[\operatorname{Ker} f_{2}\right]_{2}^{\perp}=\left\langle v_{4}\right\rangle$;

- $\operatorname{Ker} f=\left\langle v_{1}-v_{2}, v_{1}+v_{2}+v_{3}\right\rangle$ and $[\operatorname{Ker} f]^{\perp}=\left\langle v_{1}+v_{2}-2 v_{3}, v_{4}\right\rangle$.

Accordingly, $\widetilde{\mathcal{H}}_{f}^{\perp} \neq[\operatorname{Ker} f]^{\perp}$ and $\mathcal{H}_{f}^{\perp} \neq[\operatorname{Im} f]^{\perp}$ in this case. of $f$.

REMARK 3.5. Given an endomorphism $f \in \operatorname{End}_{k}(E)$, in general $f_{\mathcal{H}_{f}}^{+}$is not the Moore-Penrose inverse

EXAMPLE 3.6. If $f$ and $\mathcal{H}_{f}=\left\{H_{1}, H_{2}\right\}$ are as in Remark 3.4, a computation shows that

$$
f_{\mathcal{H}_{f}}^{+}\left(v_{i}\right)=\left\{\begin{array}{clc}
\frac{1}{2} v_{1}+\frac{1}{2} v_{2} & \text { if } & i=1 \\
v_{4}-\frac{1}{2} v_{1}-\frac{1}{2} v_{2} & \text { if } & i=3 \\
0 & \text { if } & i=2,4
\end{array}\right.
$$

and

$$
f^{\dagger}\left(v_{i}\right)=\left\{\begin{array}{cc}
\frac{1}{6} v_{1}+\frac{1}{6} v_{2}-\frac{1}{3} v_{3} & \text { if } i=1 \\
-\frac{1}{12} v_{1}-\frac{1}{12} v_{2}+\frac{1}{6} v_{3}+\frac{1}{2} v_{4} & \text { if } \quad i=2 \\
-\frac{1}{12} v_{1}-\frac{1}{12} v_{2}+\frac{1}{6} v_{3}+\frac{1}{2} v_{4} & \text { if } \quad i=3 \\
0 & \text { if } \quad i=4
\end{array} .\right.
$$

Readers can easily check that $f_{\mathcal{H}_{f}}^{+}$is a reflexive generalized inverse of $f$ (Definition 2.2), although it is clear that $f_{\mathcal{H}_{f}}^{+} \neq f^{\dagger}$.

Lemma 3.7. With the above notation, we have that $\mathcal{H}_{f}^{\perp}=[\operatorname{Im} f]^{\perp}$ if and only if

$$
\left[\operatorname{Im} f_{i}\right]_{i}^{\perp} \subseteq\left[\sum_{j \neq i} \operatorname{Im} f_{j}\right]^{\perp} \quad \text { for every } i \in\{1, \ldots, n\} .
$$

Proof. Considering that $E=\operatorname{Im} f \oplus \mathcal{H}_{f}^{\perp}$ and $[\operatorname{Im} f]^{\perp} \cap \operatorname{Im} f=\{0\}$, the statement is deduced bearing in mind that this condition is equivalent to $\mathcal{H}_{f}^{\perp} \subseteq[\operatorname{Im} f]^{\perp}$ (Lemma 3.2).

Similarly, one can prove that

LEMmA 3.8. We have that $\widetilde{\mathcal{H}}_{f}^{\perp}=[\operatorname{Ker} f]^{\perp}$ if and only if

$$
\left[\operatorname{Ker} f_{i}\right]_{i}^{\perp} \subseteq\left[\sum_{j \neq i} \operatorname{Ker} f_{j}\right]^{\perp} \quad \text { for every } i \in\{1, \ldots, n\} .
$$

Proposition 3.9. If $f \in \operatorname{End}_{k}(E)$ and $\mathcal{H}_{f}=\left\{H_{1}, \ldots, H_{n}\right\}$ is a family of subspaces of $E$ invariants for $f$ such that $E=H_{1} \oplus \cdots \oplus H_{n}$ and $H_{i} \subseteq\left[\sum_{j \neq i} H_{j}\right]^{\perp}$ for all $i \in\{1, \ldots, n\}$, then $f_{\mathcal{H}_{f}}^{+}=f^{\dagger}$.

Proof. If $H_{i} \subseteq\left[\sum_{j \neq i} H_{j}\right]^{\perp}$ for all $i \in\{1, \ldots, n\}$, then the conditions of Lemma 3.7 and Lemma 3.8 hold. 
3.2. Moore-Penrose inverse of linear maps on arbitrary inner product spaces. We shall now generalize the notion of Moore-Penrose inverse to some endomorphisms of arbitrary vector spaces, in particular some infinite-dimensional vector spaces.

Henceforth, $(V, g)$ and $(W, \bar{g})$ will be inner product vector spaces over $k$, with $k=\mathbb{C}$ or $k=\mathbb{R}$.

Definition 3.10. Given a linear map $f: V \rightarrow W$, we say that $f$ is admissible for the Moore-Penrose inverse when $V=\operatorname{Ker} f \oplus[\operatorname{Ker} f]^{\perp}$ and $W=\operatorname{Im} f \oplus[\operatorname{Im} f]^{\perp}$.

REMARK 3.11. It is known that there exist infinite-dimensional vector spaces $V$ and vector subspaces $U \subset V$ such that $V \neq U \oplus U^{\perp}$. In this case, if $V=U \oplus W$, it is clear that the linear map $f_{U} \in \operatorname{End}_{k}(V)$ defined as

$$
f_{U}(v)= \begin{cases}0 & \text { if } \quad v \in U \\ v & \text { if } \quad v \in W\end{cases}
$$

is not admissible for the Moore-Penrose inverse.

TheOREm 3.12 (Existence and uniqueness of Moore-Penrose inverse). If $(V, g)$ and $(W, \bar{g})$ are inner product spaces over $k$, then $f: V \rightarrow W$ is a linear map admissible for the Moore-Penrose inverse if and only if there exists a unique linear map $f^{\dagger}: W \rightarrow V$ such that:

1. $f^{\dagger}$ is a reflexive generalized inverse of $f$;

2. $f^{\dagger} \circ f$ and $f \circ f^{\dagger}$ are self-adjoint, that is:

- $g\left(\left[f^{\dagger} \circ f\right](v), v^{\prime}\right)=g\left(v,\left[f^{\dagger} \circ f\right]\left(v^{\prime}\right)\right.$,

- $\bar{g}\left(\left[f \circ f^{\dagger}\right](w), w^{\prime}\right)=\bar{g}\left(w,\left[f \circ f^{\dagger}\right]\left(w^{\prime}\right)\right.$

for all $v, v^{\prime} \in V$ and $w, w^{\prime} \in W$.

Proof. If $f$ is admissible of the Moore-Penrose inverse (Definition 3.10), then the restriction $f_{\left.\right|_{[\text {Ker } f] \perp}}$ is an isomorphism between $[\operatorname{Ker} f]^{\perp}$ and $\operatorname{Im} f$ and there exists a linear map satisfying that

$$
f^{\dagger}(w)=\left\{\begin{array}{cl}
\left(f_{\left.\right|_{[\operatorname{Ker} f]^{\perp}}}\right)^{-1}(w) & \text { if } \quad w \in \operatorname{Im} f \\
0 & \text { if } \quad w \in[\operatorname{Im} f]^{\perp}
\end{array} .\right.
$$

In this case, $f$ is unique.

We now check that $f^{\dagger}$ satisfies the conditions of the statement.

Firstly, since

$$
\left(f \circ f^{\dagger}\right)(w)=\left\{\begin{array}{lll}
w & \text { if } & w \in \operatorname{Im} f \\
0 & \text { if } & w \in[\operatorname{Im} f]^{\perp}
\end{array}\right.
$$

and $\left(f^{\dagger} \circ f\right)(v)=v_{1}$ with $v=v_{1}+v_{2}\left(v_{1} \in[\operatorname{Ker} f]^{\perp}\right.$ and $\left.v_{2} \in \operatorname{Ker} f\right)$, it is clear that $f^{\dagger}$ is a reflexive generalized inverse of $f$ because:

- $\left(f \circ f^{\dagger} \circ f\right)(v)=f(v)$;

- $\left(f^{\dagger} \circ f \circ f^{\dagger}\right)(w)=f^{\dagger}(w)$.

Moreover,

$$
\bar{g}\left(\left[f \circ f^{\dagger}\right](w), w^{\prime}\right)=\bar{g}\left(w,\left[f \circ f^{\dagger}\right]\left(w^{\prime}\right)\right)=\left\{\begin{array}{cl}
\bar{g}\left(w, w^{\prime}\right) & \text { if } w, w^{\prime} \in \operatorname{Im} f \\
0 & \text { if } w \in[\operatorname{Im} f]^{\perp} \\
0 & \text { if } w^{\prime} \in[\operatorname{Im} f]^{\perp}
\end{array} .\right.
$$


And, if $v, v^{\prime} \in V$ with $v=v_{1}+v_{2}, v^{\prime}=v_{1}^{\prime}+v_{2}^{\prime}, v_{1}, v_{1}^{\prime} \in[\operatorname{Ker} f]^{\perp}$ and $v_{2}, v_{2}^{\prime} \in \operatorname{Ker} f$, one has that

$$
g\left(\left[f^{\dagger} \circ f\right](v), v^{\prime}\right)=g\left(v_{1}, v_{1}^{\prime}\right)=g\left(v,\left[f^{\dagger} \circ f\right]\left(v^{\prime}\right) .\right.
$$

Hence, we conclude that $f^{\dagger}$ satisfies the conditions of the theorem.

For proving the uniqueness of the Moore-Penrose inverse of $f$, let us consider a linear map $\widetilde{f}: W \rightarrow V$ such that

1. $\tilde{f}$ is a reflexive generalized inverse of $f$,

2. $g\left([\tilde{f} \circ f](v), v^{\prime}\right)=g\left(v,[\tilde{f} \circ f]\left(v^{\prime}\right)\right.$,

3. $\bar{g}\left([f \circ \widetilde{f}](w), w^{\prime}\right)=\bar{g}\left(w,[f \circ \widetilde{f}]\left(w^{\prime}\right)\right.$

for all $v, v^{\prime} \in V$ and $w, w^{\prime} \in W$.

A direct consequence of $(1)$ is that $(\tilde{f} \circ f)^{2}=\tilde{f} \circ f$. Hence, $\tilde{f} \circ f$ is a projection and, since

$$
\operatorname{Im} f=\operatorname{Im}(f \circ \tilde{f} \circ f) \subseteq \operatorname{Im}(f \circ \widetilde{f}) \subseteq \operatorname{Im} f,
$$

then $\operatorname{Im}(f \circ \widetilde{f})=\operatorname{Im} f$.

Accordingly, given $w \in \operatorname{Im} f$, there exists $\bar{w} \in W$ such that $(f \circ \widetilde{f})(\bar{w})$, and then

$$
(f \circ \widetilde{f})(w)=(f \circ \widetilde{f})^{2}(\bar{w})=(f \circ \widetilde{f})(\bar{w})=w .
$$

Furthermore, if $w^{\prime} \in[\operatorname{Im} f]^{\perp}$, we have that

$$
0=\bar{g}\left([f \circ \widetilde{f}]^{2}\left(w^{\prime}\right), w^{\prime}\right)=\bar{g}\left([f \circ \widetilde{f}]\left(w^{\prime}\right),[f \circ \widetilde{f}]\left(w^{\prime}\right)\right) \Longrightarrow[f \circ \widetilde{f}]\left(w^{\prime}\right)=0 .
$$

Thus,

$$
(f \circ \widetilde{f})(w)=\left\{\begin{array}{lll}
w & \text { if } & w \in \operatorname{Im} f \\
0 & \text { if } & w \in[\operatorname{Im} f]^{\perp}
\end{array}\right.
$$

and, in particular, $\widetilde{f}\left(w^{\prime}\right)=0$ when $w^{\prime} \in[\operatorname{Im} f]^{\perp}$.

In line with the above arguments, one has that $(f \circ \tilde{f})^{2}=f \circ \tilde{f}$ and $\operatorname{Im}(\tilde{f} \circ f)=\operatorname{Im} \tilde{f}$.

Now, if $v \in \operatorname{Im} \tilde{f}, v=[\tilde{f} \circ f](\bar{v})$ and $v^{\prime} \in \operatorname{Ker} f$, then

$$
g\left(v, v^{\prime}\right)=g\left([\tilde{f} \circ f](\bar{v}), v^{\prime}\right)=g(\bar{v}, 0)=0,
$$

and we deduce that $\operatorname{Im} \tilde{f} \subseteq[\operatorname{Ker} f]^{\perp}$.

Finally, since $f_{\left.\right|_{[\operatorname{Ker} f]^{\perp}}}:[\operatorname{Ker} f]^{\perp} \stackrel{\sim}{\longrightarrow} \operatorname{Im} f$ and $(f \circ \widetilde{f})_{\left.\right|_{\operatorname{Im} f}}=\operatorname{Id}_{\left.\right|_{\operatorname{Im} f}}$, then

$$
(\widetilde{f})_{\mid \operatorname{Im} f}=\left(f_{\left.\right|_{[\operatorname{Ker} f]^{\perp}}}\right)^{-1} \Longrightarrow \widetilde{f}=f^{\dagger} .
$$

Conversely, let us assume that there exists the Moore-Penrose inverse $f^{\dagger}: W \rightarrow V$ of a linear map $f: V \rightarrow W$. Based on the same arguments as above one immediately has that:

- $f \circ f^{\dagger}$ and $f^{\dagger} \circ f$ are projections;

- $\operatorname{Im}\left(f \circ f^{\dagger}\right)=\operatorname{Im} f$; 
- $[\operatorname{Im} f]^{\perp} \subseteq \operatorname{Ker}\left(f \circ f^{\dagger}\right)$;

- $\operatorname{Im}\left(f^{\dagger} \circ f\right) \subseteq[\operatorname{Ker} f]^{\perp}$.

Moreover, if $w \notin[\operatorname{Im} f]^{\perp}$ there exists $\bar{w} \in W$ such that

$$
0 \neq \bar{g}\left(\left[f \circ f^{\dagger}\right]^{2}(\bar{w}), w\right)=\bar{g}\left(\left[f \circ f^{\dagger}\right](\bar{w}),\left[f \circ f^{\dagger}\right](w)\right)
$$

from where we deduce that $w \notin \operatorname{Ker}\left(f \circ f^{\dagger}\right)$ and $[\operatorname{Im} f]^{\perp}=\operatorname{Ker}\left(f \circ f^{\dagger}\right)$.

On the other hand, it is clear that $\operatorname{Ker} f \subseteq \operatorname{Ker}\left(f^{\dagger} \circ f\right)$ and, if $v \in V$ with $f(v) \neq 0$ then $v \notin \operatorname{Ker}\left(f^{\dagger} \circ f\right)$, because

$$
f(v)=\left(f \circ f^{\dagger} \circ f\right)(v) \neq 0
$$

Hence, $\operatorname{Ker} f=\operatorname{Ker}\left(f^{\dagger} \circ f\right)$ and, bearing in mind that if $g \in \operatorname{End}_{k}(V)$ is a projection then $V=$ Ker $g \oplus \operatorname{Im} g$, one concludes that

$$
V=\operatorname{Ker}\left(f^{\dagger} \circ f\right) \oplus \operatorname{Im}\left(f^{\dagger} \circ f\right)=\operatorname{Ker} f \oplus[\operatorname{Ker} f]^{\perp}
$$

and

$$
W=\operatorname{Ker}\left(f \circ f^{\dagger}\right) \oplus \operatorname{Im}\left(f \circ f^{\dagger}\right)=\operatorname{Im} f \oplus[\operatorname{Im} f]^{\perp} .
$$

Accordingly, $f$ is admissible for the Moore-Penrose inverse and the statement is deduced.

The operator $f^{\dagger}$ characterized in Theorem 3.12 is named the Moore-Penrose inverse of $f$.

Since each isomorphism $g: V \rightarrow W$ is admissible for the Moore-Penrose inverse, a direct consequence of Theorem 3.12 is that $g^{\dagger}=g^{-1}$, where $g^{-1}$ is the inverse map of $g$.

Corollary 3.13. If $(V, g)$ and $(W, \bar{g})$ are inner product spaces over $k$ and $f: V \rightarrow W$ is a linear map admissible for the Moore-Penrose inverse, then $f^{\dagger}$ is also admissible for the Moore-Penrose inverse and $\left(f^{\dagger}\right)^{\dagger}=f$.

Proof. This statement is deduced from Theorem 3.12 bearing in mind that:

- $\operatorname{Im} f^{\dagger}=[\operatorname{Ker} f]^{\perp}$

- $\left[\operatorname{Im} f^{\dagger}\right]^{\perp}=\operatorname{Ker} f$;

- $\operatorname{Ker} f^{\dagger}=[\operatorname{Im} f]^{\perp}$;

- $\left[\operatorname{Ker} f^{\dagger}\right]^{\perp}=\operatorname{Im} f$.

Moreover, if $f: V \rightarrow W$ is a linear map admissible for the Moore-Penrose inverse and $P_{[\operatorname{ker} f]}$ and $P_{\operatorname{Im} f}$ are the projections induced by the decompositions $V=\operatorname{Ker} f \oplus[\operatorname{Ker} f]^{\perp}$ and $W=\operatorname{Im} f \oplus[\operatorname{Im} f]^{\perp}$, respectively, we obtain from the arguments of the proof of Theorem 3.12 that

Corollary 3.14. If $(V, g)$ and $(W, \bar{g})$ are inner product spaces over $k$ and $f: V \rightarrow W$ is a linear map admissible for the Moore-Penrose inverse, then

- $f^{\dagger} \circ f=P_{[\operatorname{ker} f]^{\perp}}$

- $f \circ f^{\dagger}=P_{\operatorname{Im} f}$.

3.3. Computation of the Moore-Penrose inverse of endomorphisms on arbitrary inner product spaces. Similar to the finite-dimensional situation, given an inner product space $(V, g)$ over 
$k, f \in \operatorname{End}_{k}(V)$ let us assume that there exists a family of $f$-invariant finite-dimensional subspaces, $\mathcal{H}_{f}=\left\{H_{i}\right\}_{i \in I}$, such that

$$
V=\bigoplus_{i \in I} H_{i}
$$

Note that this assumption is always satisfied when $f$ admits an annihilator polynomial.

To simplify, fixing a family $\mathcal{H}_{f}$, we shall denote $f_{i}=f_{\left.\right|_{H_{i}}}$.

Definition 3.15. The unique linear map $f_{\mathcal{H}_{f}}^{+} \in \operatorname{End}_{k}(V)$ such that $\left[f_{\mathcal{H}_{f}}^{+}\right]_{H_{i}}=f_{i}^{\dagger}$ for every $i \in I$ is called the generalized inverse of $f$ associated with the family $\mathcal{H}_{f}$.

For each vector $v \in V$, if $v=v_{i_{1}}+\cdots+v_{i_{s}}$ with $v_{i_{j}} \in H_{i_{j}}$, then

$$
f_{\mathcal{H}_{f}}^{+}(v)=f_{i_{1}}^{\dagger}\left(v_{1}\right)+\cdots+f_{i_{s}}^{\dagger}\left(v_{s}\right) .
$$

If $f$ is admissible for the Moore-Penrose inverse, our purpose is to determine when $f_{\mathcal{H}_{f}}^{+}=f^{\dagger}$. To do this, the generalization onto infinite-dimensional vector spaces of Lemma 3.3 is:

Lemma 3.16. We have that:

1. $\operatorname{Im} f=\bigoplus_{i \in I} \operatorname{Im} f_{i}$;

2. If $\mathcal{H}_{f}^{\perp}=\bigoplus_{i \in I}\left[\operatorname{Im} f_{i}\right]_{i}^{\perp}$, then $V=\operatorname{Im} f \oplus \mathcal{H}_{f}^{\perp}$;

3. $\operatorname{Ker} f=\bigoplus_{i \in I} \operatorname{Ker} f_{i}$;

4. If $\widetilde{\mathcal{H}}_{f}^{\perp}=\bigoplus_{i \in I}\left[\operatorname{Ker} f_{i}\right]_{i}^{\perp}$, then $V=\operatorname{Ker} f \oplus \widetilde{\mathcal{H}}_{f}^{\perp}$;

5. $f$ induces an isomorphism between $\widetilde{\mathcal{H}}_{f}^{\perp}$ and $\operatorname{Im} f$.

Proof. 1. It is clear that $\bigoplus_{i \in I} \operatorname{Im} f_{i} \subseteq \operatorname{Im} f$. Moreover, given $v \in \operatorname{Im} f$, if $v=f\left(v^{\prime}\right)$ with $v^{\prime}=$ $v_{i_{1}}^{\prime}+\cdots+v_{i_{s}}^{\prime}$ and $v_{i_{j}}^{\prime} \in H_{i_{j}}$ for all $j \in\{1, \ldots, s\}$, then

$$
v=f\left(v_{i_{1}}^{\prime}\right)+\cdots+f\left(v_{i_{s}}^{\prime}\right) \in \bigoplus_{i \in I} \operatorname{Im} f_{i}
$$

because $f\left(v_{i_{j}}^{\prime}\right) \in H_{i_{j}}$ for every $j \in\{1, \ldots, s\}$.

2. Since $H_{i}=\operatorname{Im} f_{i} \oplus\left[\operatorname{Im} f_{i}\right]_{i}^{\perp}$ for all $i \in I$, we have that

$$
\begin{aligned}
V & =\bigoplus_{i \in I} H_{i}=\bigoplus_{i \in I}\left(\operatorname{Im} f_{i} \oplus\left[\operatorname{Im} f_{i}\right]_{i}^{\perp}\right) \\
& =\left(\bigoplus_{i \in I} \operatorname{Im} f_{i}\right) \oplus\left(\bigoplus_{i \in I}\left[\operatorname{Im} f_{i}\right]_{i}^{\perp}\right)=\operatorname{Im} f \oplus \mathcal{H}_{f}^{\perp} .
\end{aligned}
$$

3. It is immediate that $\bigoplus_{i \in I} \operatorname{Ker} f_{i} \subseteq \operatorname{Ker} f$. Furthermore, if $\bar{v} \in \operatorname{Ker} f$ and

$$
\bar{v}=\bar{v}_{i_{1}}+\cdots+\bar{v}_{i_{k}} \quad \text { with } \quad \bar{v}_{i_{j}} \in H_{i_{j}} \text { for every } j \in\{1, \ldots, k\},
$$

since

$$
f\left(\bar{v}_{i_{1}}\right)+\cdots+f\left(\bar{v}_{i_{k}}\right)=0 \text { and } H_{i_{j}} \cap\left[\sum_{r \neq j} H_{i_{r}}\right]=0,
$$

we conclude that $f\left(\bar{v}_{i_{j}}\right)=0$ for all $j \in\{1, \ldots, k\}$, from which it is deduced that $\operatorname{Ker} f \subseteq \bigoplus_{i \in I} \operatorname{Ker} f_{i}$. 
4. Similar to (2).

5. It is a direct consequence of (2) and (4).

It is now easy to prove that the generalization onto an arbitrary vector space $V$ of the Lemma 3.2 is the following:

LEMMA 3.17. With the previous assumptions on $V$ and $\mathcal{H}_{f}=\left\{U_{i}\right\}_{i \in I}$, if $U \subset V$ is a subspace and $\left\{U_{i}\right\}_{i \in I}$ is a family of subspaces of $U$ such that $U=\bigoplus_{i \in I} U_{i}$ with $U_{i} \subseteq H_{i}$, then

$$
\bigoplus_{i \in I}\left[U_{i}\right]_{i}^{\perp} \subseteq U^{\perp} \Longleftrightarrow\left[U_{i}\right]_{i}^{\perp} \subset\left[\sum_{j \neq i} U_{j}\right]^{\perp} \quad \text { for all } i \in I
$$

Accordingly, we have that:

LEMMA 3.18. If $(V, g)$ is an inner product vector space over $k, f \in \operatorname{End}_{k}(V)$, and $\mathcal{H}_{f}=\left\{H_{i}\right\}_{i \in I}$ with $V=\bigoplus_{i \in I} H_{i}$ and each $H_{i}$ is $f$-invariant, then $\mathcal{H}_{f}^{\perp}=[\operatorname{Im} f]^{\perp}$ if and only if

$$
\left[\operatorname{Im} f_{i}\right]_{i}^{\perp} \subseteq\left[\sum_{j \neq i} \operatorname{Im} f_{j}\right]^{\perp} \quad \text { for every } i \in I
$$

Proof. This statement is the generalization of Lemma 3.7 to arbitrary vector spaces.

Moreover, similar to Lemma 3.8 one has that:

LEMMA 3.19. If $(V, g)$ is an inner product vector space over $k, f \in \operatorname{End}_{k}(V)$, and $\mathcal{H}_{f}=\left\{H_{i}\right\}_{i \in I}$ with $V=\bigoplus_{i \in I} H_{i}$ and each subspace $H_{i}$ is $f$-invariant, then $\widetilde{\mathcal{H}}_{f}^{\perp}=[\operatorname{Ker} f]^{\perp}$ if and only if

$$
\left[\operatorname{Ker} f_{i}\right]_{i}^{\perp} \subseteq\left[\sum_{j \neq i} \operatorname{Ker} f_{j}\right]^{\perp} \quad \text { for every } i \in I \text {. }
$$

THEOREM 3.20. If $(V, g)$ is an inner product vector space over $k, f \in \operatorname{End}_{k}(V)$ is admissible for the Moore-Penrose inverse, and $\mathcal{H}_{f}=\left\{H_{i}\right\}_{i \in I}$ with $V=\bigoplus_{i \in I} H_{i}$ and each subspace $H_{i}$ is $f$-invariant, then $f_{\mathcal{H}_{f}}^{+}=f^{\dagger}$ if and only if the following conditions are satisfied:

1. $\left[\operatorname{Im} f_{i}\right]_{i}^{\perp} \subseteq\left[\sum_{j \neq i} \operatorname{Im} f_{j}\right]^{\perp}$ for every $i \in I$;

2. $\left[\operatorname{Ker} f_{i}\right]_{i}^{\perp} \subseteq\left[\sum_{j \neq i} \operatorname{Ker} f_{j}\right]^{\perp}$ for all $i \in I$.

Proof. The claim is a direct consequence of Lemma 3.18 and Lemma 3.19.

Corollary 3.21. If $(V, g)$ is an inner product vector space over $k, f \in \operatorname{End}_{k}(V), \mathcal{H}_{f}=\left\{H_{i}\right\}_{i \in I}$ with $V=\bigoplus_{i \in I} H_{i}$ and each subspace $H_{i}$ is $f$-invariant, and $H_{i} \subseteq\left[\sum_{j \neq i} H_{j}\right]^{\perp}$ for every $i \in I$, then $f$ is admissible for the Moore-Penrose inverse and $f_{\mathcal{H}_{f}}^{+}=f^{\dagger}$.

Proof. With the hypothesis of this Corollary the conditions of Theorem 3.20 are satisfied.

ExAmple 3.22. Let $(V, g)$ be an inner product vector space of countable dimension over $k$. Let $\left\{v_{1}, v_{2}, v_{3}, \ldots\right\}$ be an orthonormal basis of $V$ indexed by the natural numbers. 
Electronic Journal of Linear Algebra, ISSN 1081-3810

Let $\varphi \in \operatorname{End}_{k}(V)$ the finite potent endomorphism defined as follows:

$$
\varphi\left(v_{i}\right)=\left\{\begin{array}{cll}
v_{2}+v_{5}+v_{7} & \text { if } \quad i=1 \\
v_{1}+3 v_{2} & \text { if } \quad i=2 \\
v_{4} & \text { if } \quad i=3 \\
v_{1}-v_{3} & \text { if } \quad i=4 \\
-v_{3}+2 v_{5}+2 v_{7} & \text { if } \quad i=5 \\
3 v_{i+1} & \text { if } \quad i=5 h+1 \\
0 & \text { if } \quad i=5 h+2 \\
-v_{i-2}+2 v_{i+1} & \text { if } \quad i=5 h+3 \\
v_{i-2}+v_{i+1} & \text { if } \quad i=5 h+4 \\
-v_{i-4}+5 v_{i-3} & \text { if } & i=5 h+5
\end{array}\right.
$$

for all $h \geq 1$.

We can consider the decomposition $V=\bigoplus_{i \in \mathbb{N}} H_{i}$ where

$$
H_{1}=\left\langle v_{i}\right\rangle_{i \in\{1, \ldots, 10\}} \quad \text { and } \quad H_{j}=\left\langle v_{5 j+1}, v_{5 j+2}, v_{5 j+3}, v_{5 j+4}, v_{5 j+5}\right\rangle
$$

for every $j \geq 2$. It is clear that $H_{i}$ is a $\varphi$-invariant subspace of $V$ for every $i \in \mathbb{N}$.

In the above bases one has that

$$
\varphi_{\left.\right|_{H_{1}}} \equiv\left(\begin{array}{cccccccccc}
0 & 1 & 0 & 1 & 0 & 0 & 0 & 0 & 0 & 0 \\
1 & 3 & 0 & 0 & 0 & 0 & 0 & 0 & 0 & 0 \\
0 & 0 & 0 & -1 & -1 & 0 & 0 & 0 & 0 & 0 \\
0 & 0 & 1 & 0 & 0 & 0 & 0 & 0 & 0 & 0 \\
1 & 0 & 0 & 0 & 2 & 0 & 0 & 0 & 0 & 0 \\
0 & 0 & 0 & 0 & 0 & 0 & 0 & -1 & 0 & -1 \\
0 & 0 & 0 & 0 & 0 & 0 & 0 & 0 & 0 & 0 \\
0 & 0 & 0 & 0 & 0 & 0 & 0 & 0 & 0 & 0 \\
0 & 0 & 0 & 0 & 0 & 0 & 0 & 2 & 0 & 0 \\
0 & 0 & 0 & 0 & 0 & 0 & 0 & 0 & 1 & 0
\end{array}\right)
$$

and

$$
\varphi_{\left.\right|_{H_{i}}} \equiv\left(\begin{array}{ccccc}
0 & 0 & -1 & 0 & -1 \\
3 & 0 & 0 & 1 & 5 \\
0 & 0 & 0 & 0 & 0 \\
0 & 0 & 2 & 0 & 0 \\
0 & 0 & 0 & 1 & 0
\end{array}\right)
$$

for all $i \geq 2$.

Thus, bearing in mind that:

- $\operatorname{Im} \varphi=\left\langle v_{1}, v_{2}, v_{3}, v_{4}, v_{5}\right\rangle \oplus\left[\underset{i \geq 1}{\oplus}\left\langle v_{5 j+1}, v_{5 j+2}, v_{5 j+4}, v_{5 j+5}\right\rangle\right]$ and $[\operatorname{Im} \varphi]^{\perp}=\underset{i \geq 1}{\oplus}\left\langle v_{5 i+3}\right\rangle$;

- $\operatorname{Ker} \varphi=\underset{i \geq 1}{\oplus}\left\langle v_{5 i+2}\right\rangle$ and $[\operatorname{Ker} \varphi]^{\perp}=\left\langle v_{1}, v_{2}, v_{3}, v_{4}, v_{5}\right\rangle \oplus\left[\underset{i \geq 1}{\oplus}\left\langle v_{5 j+1}, v_{5 j+3}, v_{5 j+4}, v_{5 j+5}\right\rangle\right]$; 
Electronic Journal of Linear Algebra, ISSN 1081-3810

we have that $\varphi$ is admissible for the Moore-Penrose inverse and, since $\left\{v_{i}\right\}_{i \in I}$ is an orthonormal basis of $V$, Corollary 3.21 holds for $\varphi$.

Accordingly, a non-difficult computation shows that

$$
\left(\varphi^{\dagger}\right)_{\left.\right|_{1}} \equiv\left(\begin{array}{cccccccccc}
6 & -2 & 6 & 0 & 3 & 0 & 0 & 0 & 0 & 0 \\
-2 & 1 & -2 & 0 & -1 & 0 & 0 & 0 & 0 & 0 \\
0 & 0 & 0 & 1 & 0 & 0 & 0 & 0 & 0 & 0 \\
3 & -1 & 2 & 0 & 1 & 0 & 0 & 0 & 0 & 0 \\
-3 & 1 & -3 & 0 & -1 & 0 & 0 & 0 & 0 & 0 \\
0 & 0 & 0 & 0 & -\frac{1}{3} & \frac{5}{3} & \frac{1}{3} & 0 & \frac{5}{6} & -\frac{1}{3} \\
0 & 0 & 0 & 0 & 0 & 0 & 0 & 0 & 0 & 0 \\
0 & 0 & 0 & 0 & 0 & 0 & 0 & 0 & \frac{1}{2} & 0 \\
0 & 0 & 0 & 0 & 0 & 0 & 0 & 0 & 0 & 1 \\
0 & 0 & 0 & 0 & 0 & -1 & 0 & 0 & -\frac{1}{2} & 0
\end{array}\right)
$$

and

$$
\left(\varphi^{\dagger}\right)_{\left.\right|_{H_{i}}} \equiv\left(\begin{array}{ccccc}
\frac{5}{3} & \frac{1}{3} & 0 & \frac{5}{6} & -\frac{1}{3} \\
0 & 0 & 0 & 0 & 0 \\
0 & 0 & 0 & \frac{1}{2} & 0 \\
0 & 0 & 0 & 0 & 1 \\
-1 & 0 & 0 & -\frac{1}{2} & 0
\end{array}\right)
$$

for all $i \geq 2$, from where the endomorphism $\varphi^{\dagger}$ is determined.

Thus, the explicit expression of $\varphi^{\dagger}$ is

$$
\varphi^{\dagger}\left(v_{i}\right)=\left\{\begin{array}{cl}
6 v_{1}-2 v_{2}+3 v_{4}-3 v_{5}-3 v_{7} & \text { if } i=1 \\
-2 v_{1}+v_{2}-v_{4}+v_{5}+v_{7} & \text { if } i=2 \\
6 v_{1}-2 v_{2}+2 v_{4}-3 v_{5}-3 v_{7} & \text { if } i=3 \\
v_{3} & \text { if } i=4 \\
3 v_{1}-v_{2}+v_{4}-v_{5}-\frac{1}{3} v_{6}-v_{7} & \text { if } i=5 \\
\frac{5}{3} v_{i}-v_{i+4} & \text { if } i=5 h+1 \\
\frac{1}{3} v_{i-1} & \text { if } i=5 h+2 \\
0 & \text { if } i=5 h+3 \\
\frac{1}{6} v_{i-3}+\frac{1}{2} v_{i-1}-\frac{1}{2} v_{i+1} & \text { if } i=5 h+4 \\
-\frac{1}{3} v_{i-4}+v_{i-1} & \text { if } i=5 h+5
\end{array}\right.
$$

for all $h \geq 1$.

Remark 3.23. We wish point out that this example shows that the Moore-Penrose inverse of a finite potent endomorphism is not, in general, a finite potent endomorphism. 
4. Study of infinite systems of linear equations from the Moore-Penrose inverse. The aim of this final section is to study solutions of infinite system of linear equations from the Moore-Penrose inverse of linear maps characterized in the previous section.

Definition 4.1. If $V$ and $W$ are two arbitrary $k$-vector spaces and $f: V \rightarrow W$ is a linear map, a linear system is an expression

$$
f(x)=w
$$

where $w \in W$. This system is called "consistent" when $w \in \operatorname{Im} f$.

If $V$ and $W$ are infinite-dimensional vector spaces, fixing bases of $V$ and $W$, the linear system $f(x)=w$ is equivalent to an infinite system of linear equations.

If a linear system $f(x)=w$ is consistent and $f\left(v_{0}\right)=w$ for a certain $v_{0} \in V$, then the set of solutions of this system is $v_{0}+\operatorname{Ker} f$. The vector $v_{0}$ is named "particular solution" of the system.

Let us now consider two arbitrary inner product spaces $(V, g)$ and $(W, \bar{g})$ over $k$, let $f: V \rightarrow W$ be a linear map admissible for the Moore-Penrose inverse and let $f^{\dagger}$ be its Moore-Penrose inverse. If $f(x)=w$ is a linear system, since $f^{\dagger}$ is a reflexive generalized inverse of $f$, then $f$ is consistent if and only if $\left(f \circ f^{\dagger}\right)(w)=w$ and, in this case, the set of solutions of the linear system is

$$
f^{\dagger}(w)+\operatorname{Ker} f
$$

In finite-dimensional inner product vector spaces, it is known that the Moore-Penrose inverse is useful for studying the least squares solutions of a linear system. To complete this work, we shall generalize this notion to arbitrary vector spaces.

Definition 4.2. If $(V, g)$ and $(W, \bar{g})$ are two arbitrary inner product spaces over $k$, then $v^{\prime} \in V$ is called "least $\bar{g}$-norm solution" of a linear system $f(x)=w$ when

$$
\left\|f\left(v^{\prime}\right)-w\right\|_{\bar{g}} \leq\|f(v)-w\|_{\bar{g}}
$$

for all $v \in V$.

Note that $v^{\prime} \in V$ is a least $\bar{g}$-norm solution of the linear system $f(x)=w$ if and only if $d_{\bar{g}}\left(w, f\left(v^{\prime}\right)\right)=$ $d_{g}(w, \operatorname{Im} f)$.

Definition 4.3. If $(V, g)$ and $(W, \bar{g})$ are two arbitrary inner product spaces over $k$, then $\tilde{v} \in V$ is called "minimal least $\bar{g}$-norm solution" of a linear system $f(x)=w$ when

$$
\|\tilde{v}\|_{g} \leq\left\|v^{\prime}\right\|_{g}
$$

for every least $\bar{g}$-norm solution $v^{\prime} \in V$.

Proposition 4.4. If $(V, g)$ and $(W, \bar{g})$ are two arbitrary inner product spaces over $k, f: V \rightarrow W$ is a linear map admissible for the Moore-Penrose inverse and $f(x)=w$ is a linear system, then $f^{\dagger}(w)$ is the unique minimal least $\bar{g}$-norm solution of this linear system.

Proof. Firstly, since $\operatorname{Im}\left(f \circ f^{\dagger}-\operatorname{Id}\right) \subseteq[\operatorname{Im} f]^{\perp}$, one has that

$$
\begin{aligned}
\|f(v)-w\|_{\bar{g}}^{2} & =\left\|\left[f(v)-f\left(f^{\dagger}(w)\right)\right]+\left[f\left(f^{\dagger}(w)\right)-w\right]\right\|_{\bar{g}}^{2} \\
& =\left\|f(v)-f\left(f^{\dagger}(w)\right)\right\|_{\bar{g}}^{2}+\left\|f\left(f^{\dagger}(w)\right)-w\right\|_{\bar{g}}^{2}
\end{aligned}
$$


for all $v \in V$. Hence,

$$
\left\|f\left(f^{\dagger}(w)\right)-w\right\|_{\bar{g}} \leq\|f(v)-w\|_{\bar{g}}
$$

for all $v \in V$, and we deduce that $f^{\dagger}(w)$ is a least $\bar{g}$-norm solution of $f(x)=w$.

Moreover, it follows from (4.7) that $v^{\prime} \in V$ is a least $\bar{g}$-norm solution of this linear system if and only if $f\left(v^{\prime}\right)-f\left(f^{\dagger}(w)\right)=0$, that is, $v^{\prime}$ is a solution of the consistent system

$$
f(x)-f\left(f^{\dagger}(w)\right)=0
$$

Thus, for each least $\bar{g}$-norm solution $v^{\prime} \in V$, one has that

$$
f^{\dagger}(w)=v^{\prime}+h
$$

with $h \in \operatorname{Ker} f$ and, bearing in mind that $f^{\dagger}(w) \in[\operatorname{Ker} f]^{\perp}$, we conclude that $f^{\dagger}(w)$ is the unique minimal least $\bar{g}$-norm solution of $f(x)=w$ because

$$
\left\|f^{\dagger}(w)\right\|_{g}<\left\|v^{\prime}\right\|_{g}
$$

for every $v^{\prime} \neq f^{\dagger}(w)$.

EXAMPLE 4.5. Let $(V, g)$ be an inner product space of countable dimension over $k$. Let $\left\{v_{1}, v_{2}, v_{3}, \ldots\right\}$ be an orthonormal basis of $V$ indexed by the natural numbers. If $\left(x_{i}\right)_{i \in \mathbb{N}} \in \underset{i \in \mathbb{N}}{\bigoplus} k$, since $x_{i}=0$ for almost all $i \in \mathbb{N}$, we shall write $x=\left(x_{i}\right)$ to denote the well-defined vector

$$
x=\sum_{i \in \mathbb{N}} x_{i} \cdot v_{i} \in V .
$$

Let $\varphi \in \operatorname{End}_{k}(V)$ the finite potent endomorphism studied in Example 3.22. We can consider the system

$$
\varphi(x)=w
$$

where $w=\left(\alpha_{i}\right)_{i \in \mathbb{N}}$ and whose explicit expression is:

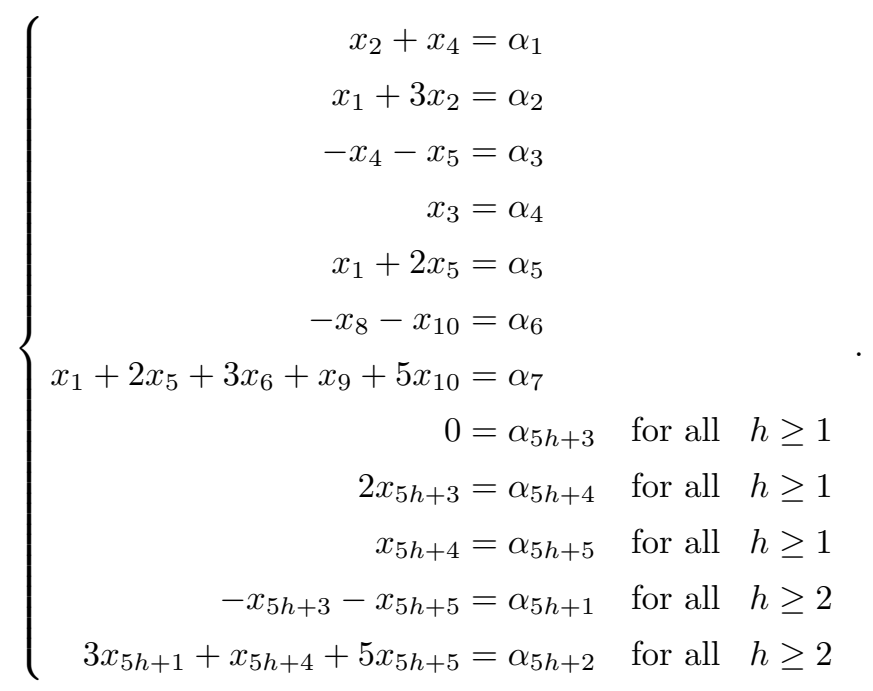


By Proposition 4.4, we have that the unique minimal least $g$-norm solution of this linear system is $\left(\beta_{i}\right)_{i \in \mathbb{N}}=\varphi^{\dagger}(w)$ and, bearing in mind the expression of $\varphi^{\dagger}$ obtained in Example 3.22, an easy computation shows that

$$
\beta_{i}=\left\{\begin{array}{cl}
6 \alpha_{1}-2 \alpha_{2}+6 \alpha_{3}+3 \alpha_{5} & \text { if } i=1 \\
-2 \alpha_{1}+\alpha_{2}-2 \alpha_{3}-\alpha_{5} & \text { if } i=2 \\
\alpha_{4} & \text { if } i=3 \\
3 \alpha_{1}-\alpha_{2}+2 \alpha_{3}+\alpha_{5} & \text { if } i=4 \\
-3 \alpha_{1}+\alpha_{2}-3 \alpha_{3}-\alpha_{5} & \text { if } i=5 \\
-\frac{1}{3} \alpha_{5}+\frac{5}{3} \alpha_{6}+\frac{1}{3} \alpha_{7}+\frac{5}{6} \alpha_{9}-\frac{1}{3} \alpha_{10} & \text { if } i=6 \\
0 & \text { if } i=7 \\
\frac{1}{2} \alpha_{i+1} & \text { if } i=5 h+3, \text { for all } \mathrm{h} \geq 1 \\
\alpha_{i+1} & \text { if } i=5 h+4, \text { for all } \mathrm{h} \geq 1 \\
-\alpha_{i-4}-\frac{1}{2} \alpha_{i-1} & \text { if } i=5 h+5, \text { for all } \mathrm{h} \geq 1 \\
\frac{1}{3} \alpha_{i+1}+\frac{5}{6} \alpha_{i+3}-\frac{1}{3} \alpha_{i+4} & \text { if } i=5 h+1, \text { for all } \mathrm{h} \geq 2 \\
0 & \text { if } i=5 h+2, \text { for all } \mathrm{h} \geq 2
\end{array} .\right.
$$

Moreover, one have that

$$
\left(\varphi \circ \varphi^{\dagger}\right)\left(v_{i}\right)=\left\{\begin{array}{rl}
0 & \text { if } i=5 h+3 \\
v_{i} & \text { otherwise }
\end{array} .\right.
$$

Thus, according to (2.4), the system (4.8) is consistent if and only if $\alpha_{5 h+3}=0$ for all $h \geq 1$, and, in this case, $\left(\beta_{i}\right)_{i \in \mathbb{N}}$ is a particular solution of it.

\section{REFERENCES}

[1] M. Argerami, F. Szechtman, and R. Tifenbach. On Tate's trace. Linear and Multilinear Algebra, 55(6):515-520, 2007.

[2] S.L. Campbell and C.D. Meyer. Generalized Inverses of Linear Transformations. Dover Publications, 1991.

[3] V. Cabezas Sánchez and F. Pablos Romo. Explicit solutions of infinite systems of linear equations from reflexive generalized inverses of finite potent endomorphisms. Linear Algebra and its Applications, 559:125-144, 2018.

[4] F. Pablos Romo. On the Drazin inverse of finite potent endomorphisms. Linear and Multilinear Algebra, 67(10):2135-2146, 2019.

[5] F. Pablos Romo. On the classification of endomorphisms on infinite-dimensional vector spaces. Georgian Mathematical Journal, DOI:10.1515/gmj-2020-2054, 2020.

[6] C.R. Rao and S.K. Mitra. Generalized Inverse of Matrices and its Applications. John Wiley \& Sons, New York, 1971.

[7] J. Tate. Residues of differentials on curves. Annales scientifiques de l'École Normale Supérieure, Serie 4, 1(1):149$159,1968$. 